\title{
Evaluación de las Habilidades del Pensamiento Crítico con la mediación de las TIC, en contextos de educación media
}

\author{
Jaime Jehosua Júdex-Orcasitas - Universidad del Norte \\ (D) $0000-0001-8095-3669$ \\ Mónica Patricia Borjas - Universidad del Norte \\ (i) $0000-0001-7823-477 \mathrm{X}$ \\ Evelin Sofía Torres Saldaña - Universidad del Norte \\ 0000-0002-3667-7369
}

Recepción: 20.12.2018 | Aceptado: 08.01.2019

Correspondencia a través de ORCID: Jaime Jehosua Júdex

iD 0000-0001-8095-3669

Citar: Júdex-Orcasitas, JJ., Borjas, MP. y Torres Saldaña, ES. (2019).Evaluación de las habilidades del pensamiento crítico con la mediación de las TIC, en contextos de educación media. REIDOCREA, 8, 21-34. [ ]

Resumen: El desarrollo del pensamiento crítico es una de las principales preocupaciones de los sistemas educativos actuales. Este se explica como la capacidad que tiene el ser humano de cuestionar su propio pensamiento y el de los demás. El objetivo de este artículo es presentar la evaluación como oportunidad formativa para movilizar el pensamiento crítico de los estudiantes de secundaria en el área de Ciencias Naturales, específicamente en el componente de habilidades. Las actividades evaluativas fueron desarrolladas mediante EDMODO. Los resultados evidencian que las actividades de evaluación favorecieron algunas categorías del componente habilidades del pensamiento crítico; sin embargo exige un largo plazo, en categorías de resolución de problemas y razonamiento escrito/verbal porque no se evidenciaron avances considerables. Esta investigación, aporta orientaciones pedagógicas para ubicar la acción evaluativa como aliada en la potenciación de habilidades de un nivel de pensamiento superior como lo es el pensamiento crítico.

Palabras clave: Pensamiento crítico | TIC

Assessment of Critical Thinking Skills Using ICT in the Context of Secondary Education

Abstract: The development of critical thinking is one of the main concerns of the educational systems nowadays. This is explained as the ability of human beings to question, not only their own thinking, but also that of others. The aim of this article is to present assessment as a formative opportunity to enhance the critical thinking of high school students in the Natural Sciences area, specifically in the skill component. The assessment activities were carried out using EDMODO and the results show that the assessment activities favored some categories of the critical thinking skills component. Nevertheless, a longer period is required in the problem solving and written/verbal reasoning categories since considerable progress was not demonstrated. This research study provides pedagogical guidelines to position assessment as an aid in the enhancement of the skills of a superior reasoning level, such as critical thinking.

Keywords: Critical Thinking | ICT

\section{Introducción}

Uno de los principales intereses de la educación formal se centra en la promoción del pensamiento crítico de los estudiantes, por lo cual resulta importarte traer a la escuela las habilidades, la disposición y la motivación que poseen los jóvenes al argumentar o discutir sobre los temas que les interesan. Es fácil escucharlos comentar sobre deportes, programas de televisión, sobre sus artistas o cuestiones que no son aquellas que reclama el mundo del conocimiento científico. También pueden argumentar con profundidad, viendo alternativas y tomando una posición clara con respecto a algo. Cómo orientar esas habilidades del pensamiento y del discurso hacia temas del aula, es problema de las aulas y de los profesores de hoy día.

Propuestas a nivel internacional atienden este interés, como la ATC21S (por sus siglas en inglés: Assessment and Teaching of 21 Century Skills) que enfatiza en la promoción del pensamiento crítico, la resolución de problemas y la toma de decisiones 
como competencias esenciales del siglo XXI (Severin, 2011). Del mismo modo, se resalta el Marco P21 para el Aprendizaje del Siglo 21 (Partnership for 21st Century Skills), que propone este tipo de pensamiento, junto con las habilidades como la creatividad, la comunicación y la colaboración como ejes transversales en la educación formal (Bellanca y Brandt, 2010).

Desde una perspectiva tradicional el pensamiento crítico, se define como un componente fundamental de la cognición, asociándolo a la argumentación y su relación con el contexto en el que esta ocurre (Bailin, Case, Coombs, y Daniels, 1999; Drewett, 1995; Ennis, 1993). Más allá de esta perspectiva, diferentes autores le conceden el atributo de mejorar la calidad del pensamiento, al apoderarse de las estructuras inherentes del acto de pensar y al someterlas a estándares intelectuales (Lipman, 1997; Paul y Elder, 2003; Saiz y Rivas, 2008; Siegel, 1985). En este sentido la metacognición proporciona el feedback que orienta el saber pensar críticamente (Valenzuela y Nieto, 2009).

Según el modelo propuesto por Halpern (1998), el pensamiento crítico está integrado por cuatro componentes: habilidades, disposiciones, transferencia (enseñanza) y monitoreo metacognitivo. De acuerdo a esta autora, las habilidades se consideran destrezas cognitivas que el pensador reconoce y utiliza de forma adecuada al momento de enfrentarse a una situación. Autores como Díaz-Barriga (2001); Duran y Sendag (2012); Olivares y Heredia (2012) plantean que dichas habilidades se relacionan directamente con el aprendizaje de los estudiantes, lo cual implica por parte del docente el diseño e implementación en el aula de estrategias que potencien dichas capacidades cognitivas permitiéndole que a través de procesos de reflexión crítica, contraste lo que sabe con hechos de la realidad.

Algunas de las habilidades que Halpern (1998) identifica como asociadas al pensamiento crítico son: el razonamiento verbal y escrito, es decir la capacidad de comprender y utilizar la información que está incluida en nuestro lenguaje cotidiano; el análisis del argumento que permite identificar información relevante, supuestos y creencias; la formulación de hipótesis; la posibilidad de analizar una situación identificando imprecisiones, contradicciones o reconociendo las limitaciones y de la ejecutoria de planes de contingencia, así como la resolución de problemas utilizando múltiples estrategias que pueden implementarse para analizar y resolver un problema de la vida real. Por su parte autores como Paul y Elder (2003), Enis (1993), Facione (1990), y López (2012), identifican como habilidades de pensamiento crítico aquellas que requieren una carga cognitiva alta como la interpretación, el análisis de situaciones, inferencia, argumentación, evaluación de diferentes situaciones, el auto monitoreo y la metacognición. Para el presente trabajo se tomó como referencia la propuesta de Halpern (1998; 2003; 2006).

Para incentivar el desarrollo del pensamiento crítico en el aula, los docentes pueden implementar diversas estrategias como: análisis de casos, debates, técnicas grupales presenciales en el marco del aprendizaje activo (Rumpagaporn, 2007). Las tecnologías de la información y la comunicación (TIC) pueden constituirse en mediaciones pedagógicas para la promoción de este tipo de pensamiento (Thompson y Crompton, 2010; Robles y Rodríguez, 2013; Akyüz, Samsa y Keser, 2015; Sá, 2016).

Promover el pensamiento crítico, exige su continua valoración y evaluación. Este implica la habilidad de evaluar desde valoraciones éticas y científicas la realidad para proponer una acción ante esta, examinando, decidiendo y defendiendo lo que se propone. Modelos de evaluación del pensamiento crítico, como el Ennis- Weir test (Ennis y Weir, 1985), El Cornell Critical Thinking Test (Ennis et. al, 1964) y el Illinois 
Critical Thinking Essay Test (Ennis y Finken, 1993), resultan pertinentes como medio de valoración argumentativa de los aprendizajes. Propuestas evaluativas como el test "California Critical Thinking Disposicional Inventory - CCTDI" (Facione, Facione, Blohm y Gittens, 1990; Facione, Facione y Giancarlo, 2001) se especializan en medir las disposiciones de este pensamiento.

En este punto la evaluación del pensamiento crítico mediada por TIC puede aprovechar los contextos variados, reales y retadores que la red brinda. Autores como Calle Álvarez (2014) y Wichadee (2014) y DeNoyelles y Reyes-Foster (2015), consideran que las TIC actúan como mediadoras que permiten que los estudiantes se motiven y centren su interés en las tareas asignadas, aumentando su compromiso en ellas a medida que estos empiezan a pensar críticamente. Los ambientes que se asemejen a las redes sociales o cuya interfaz opere igual, se vuelven una opción válida a la hora de articular las TIC y el pensamiento crítico dado que estas aumentan la motivación de los estudiantes (López Silva et al. 2016). EDMODO al ser una red social netamente académica cumple con este fin, dado que se puede mediar entre las habilidades de pensamiento crítico y el contexto real del estudiante (Cano y González, 2017). A su vez, esta plataforma favorece la producción escrita, el tratamiento de la información, el trabajo colaborativo y la autogestión, el desarrollo de la autonomía en el uso de herramientas diversas y la dinamización de la relación docente-estudiante (Duque, 2015; Vásquez Cano, 2013).

\section{Objetivos}

Comprender cómo las estrategias de evaluación formativa mediadas por TIC, posibilitan el desarrollo de las habilidades del pensamiento crítico en estudiantes de educación media en el área de ciencias naturales

\section{Método}

Esta investigación es de corte cualitativo, enfocada en comprender la dinámica de evaluación del pensamiento crítico bajo el componente de habilidades. Teniendo en cuenta este propósito se optó por el diseño de estudio de caso intrínseco (Stake, 2007). El caso presente fue un grupo de estudiantes de décimo grado, matriculados en la asignatura de Biología Avanzada de una Institución educativa de carácter oficial del área metropolitana de Barranquilla. Los principales criterios de selección del caso fueron: que la institución estuviese aprobada por el Ministerio de Educación Nacional Colombiano y contar con el aval de los directivos de la institución.

\section{Participantes}

De acuerdo al caso mencionado, los participantes fueron 32 estudiantes del grado décimo de educación media, de edades entre los 14 y 16 años y un (1) docente encargado de la asignatura de Biología avanzada, los estudiantes fueron pertenecientes a un grupo asignado a la carga académica del docente investigador.

\section{Etapas}

Para desarrollar la presente investigación se tuvieron en cuenta las siguientes etapas:

- Fundamentación: se realizó la revisión de la literatura relacionada con las categorías de Pensamiento crítico, evaluación y las TIC en educación. El producto de esta fase fue la definición de indicadores que posibilitaron la elaboración de los instrumentos: a) Guía para revisión documental y grupo focal y b) cuestionario para 
la evaluación del pensamiento crítico teniendo en cuenta los referentes de Halpern (1998; 2003; 2006). Igualmente, se seleccionó la plataforma educativa EDMODO porque permite la comunicación sincrónica y asincrónica, flexibilidad de horarios, aprendizaje colaborativo y se convierte en un ambiente propicio para dinamizar los roles de los estudiantes y el docente.

- Acercamiento a la realidad: aquí se hizo la presentación del proyecto al cuerpo directivo de la institución y a los estudiantes participantes, se obtuvo el consentimiento informado para el desarrollo de cada una de las actividades y se inició la capacitación a los estudiantes participantes en el uso de la plataforma EDMODO.

- Diseño: se diseñaron las actividades que permitieron la valoración del pensamiento crítico en el contexto de los contenidos estipulados por el ministerio de educación nacional en la asignatura de Biología Avanzada (Ministerio de Educación Nacional, 2006). Se diseñaron en total 4 actividades las cuales fueron ingresadas en el curso creado en EDMODO. El formato de diseño de cada actividad evaluativa incluye una explicación de las respectivas asignaciones o retos que deberían desarrollar los estudiantes a manera de evaluación de la asignatura. La actividad No. 1 titulada “¿Quién soy?, ¿de dónde vengo?”, contenía cinco (5) asignaciones planificadas para desarrollarse en un tiempo aproximado de 4 horas. La segunda actividad evaluativa "¿Quién manda a quién?: ¿Genes o entorno?" con seis (6) asignaciones fue diseñada para resolverse en aproximadamente 5 horas y la actividad $\mathrm{N}^{\circ} 3$ "Los Melanesios, la raza humana más nueva vinculada con extraterrestres" con seis (6) asignaciones para ser resueltas en 5 horas. Estas actividades fueron desarrolladas por los estudiantes en sus casas a manera de trabajo independiente con retroalimentación constante del profesor.

- Aplicación: Iniciando esta etapa los estudiantes, vía web, realizaron una autoevaluación PRE (cuestionario de auto-reporte), con 12 ítems del componente de Habilidades del Pensamiento Crítico. Los estudiantes también crearon sus usuarios para tener acceso al curso en EDMODO y los investigadores subieron al curso las actividades evaluativas diseñadas. Los estudiantes desarrollaron las actividades propuestas y el profesor de la asignatura brindaba retroalimentación constante en la clase presencial y a través de la plataforma. Una vez finalizado el proceso se aplicó nuevamente el instrumento de autoevaluación POST del componente Habilidades del Pensamiento Crítico. Al finalizar esta etapa, se realizó un grupo focal en el que participaron 10 estudiantes para conocer las fortalezas, debilidades y el desempeño en general del desarrollo de los actividades por parte de las estudiantes.

- Análisis de resultados y discusión: Los investigadores realizaron una revisión documental de las actividades evaluativas apoyados de una matriz que posibilitó analizar las evidencias consignadas por los estudiantes. En esta matriz se tuvo en cuenta las categorías del componente de Habilidades del pensamiento crítico propuestos por Halpern (1998; 2003; 2006). También se realizó la triangulación de los resultados de la autoevaluación PRE y POST (autorreportes del diagnóstico inicial y final), el grupo focal y la revisión documental. Para la categorización de la información derivada de las últimas técnicas mencionadas se utilizó la versión 11 del software de análisis cualitativo NVIVO Pro.

\section{Resultados}

A continuación se presentan los resultados los cuales se han organizado alrededor de las categorías asociadas al componente de Habilidades del Pensamiento Crítico: a) razonamiento escrito y verbal, b) análisis del argumento, c) resolución de problemas, c) formulación de hipótesis y d) probabilidad e incertidumbre. 
El análisis de los datos, evidencia que cada una de estas categorías presentó comportamientos diferentes. Para el caso de la categoría de razonamiento escrito $y$ verbal, se evidenció que al comparar los resultados de la autoevaluación inicial (Pre) con los de la autoevaluación final (Post), los estudiantes reconocieron que las estrategias de evaluación propuestas con la mediación de las TIC les permitieron mejorar el reconocimiento de la intención del autor y la utilización del lenguaje técnico en las actividades propuestas (ver tabla 1).

\begin{tabular}{|c|c|c|c|c|c|c|}
\hline Indicadores & Pre-siempre & Post-siempre & Pre-algunas veces & Post-algunas veces & Prenunca & Post-nunca \\
\hline Reconoce intención autor & $21,9 \%$ & $27,6 \%$ & $75 \%$ & $72,4 \%$ & $3,1 \%$ & $0 \%$ \\
\hline Utiliza lenguaje técnico & $15,6 \%$ & $27,6 \%$ & $81,3 \%$ & $72,4 \%$ & $3,1 \%$ & $0 \%$ \\
\hline Reconoce errores & $18,8 \%$ & $10,3 \%$ & $81,3 \%$ & $79,3 \%$ & $0 \%$ & $10,3 \%$ \\
\hline Emite juicios & $37,5 \%$ & $65,5 \%$ & $59,4 \%$ & $31 \%$ & $3,1 \%$ & $3,4 \%$ \\
\hline
\end{tabular}

Los datos del grupo focal y de la revisión documental coinciden en gran parte con la apreciación de los estudiantes. No obstante, el uso del lenguaje técnico de manera reiterada fue percibido por parte de los estudiantes como una dificultad, como se muestra a continuación: Estudiante YC (Grupo focal) - "bueno lo que más se me dificultó fueron las palabras desconocidas, porque a veces tenía que ver mucho con la pregunta y debía saber cuáles eran las palabras desconocidas"

Revisando los desarrollos de los estudiantes en las actividades de evaluación, se observa que al final del proceso, un gran porcentaje de estudiantes $(50 \%$ alta frecuencia y $25 \%$ frecuencia ocasional) evidencian un manejo pertinente de una terminología técnica propia de la asignatura, tal como se aprecia en la evidencia de la última actividad de evaluación: Estudiante GR (Revisión documental) - "como dice la tercera ley de Mendel, -hay rasgos que se obtiene de forma independiente sin relación con el fenotipo, y que esto no afecta al patrón de herencia de otros rasgos -, posteriormente de haber leído el artículo encuentro que según este, existe una variante única que solo afecta a dicho grupo en concreto, entonces la ley tercera de Mendel se cumpliría, ya que esta variante se desarrolló de formas independiente".

En la categoría razonamiento escrito/verbal, el indicador relacionado con reconocimiento de errores presenta en la autoevaluación un comportamiento negativo como se reflejó en la tabla 1 . No obstante, en la revisión documental realizada este indicador se presenta como fortaleza. Algunos estudiantes a lo largo de las actividades pensaban que se les había dificultado encontrar errores en los planteamientos de otros compañeros o del autor de los textos o recursos educativos incluidos en las actividades evaluativas. Sin embargo, la revisión documental del desarrollo de las actividades evaluativas da cuenta de evidencias de este indicador como se muestra en la tabla 2.

Tabla 2. Resultados Revisión documental de la categoría Razonamiento escrito/verbal

\begin{tabular}{|c|c|c|c|c|c|c|c|c|c|}
\hline \multirow[b]{3}{*}{ Indicador } & \multicolumn{9}{|c|}{ Frecuencia del hallazgo } \\
\hline & \multicolumn{3}{|c|}{ Siempre } & \multicolumn{3}{|c|}{ Algunas veces } & \multicolumn{3}{|c|}{ Nunca } \\
\hline & \multicolumn{3}{|c|}{$\mathrm{N}^{\circ}$ Actividad } & \multicolumn{3}{|c|}{$\mathrm{N}^{\circ}$ Actividad } & \multicolumn{3}{|c|}{$\mathrm{N}^{\circ}$ Actividad } \\
\hline & I & II & III & I & II & III & I & II & III \\
\hline Reconoce intención autor & $89 \%$ & $62.5 \%$ & $67 \%$ & $11 \%$ & $18.7 \%$ & $21 \%$ & $0 \%$ & $18.7 \%$ & $12 \%$ \\
\hline Utiliza lenguaje técnico & $27 \%$ & $47 \%$ & $50 \%$ & $46 \%$ & $37.5 \%$ & $25 \%$ & $27 \%$ & $15.6 \%$ & $25 \%$ \\
\hline Reconoce errores & $81 \%$ & $37.5 \%$ & $79 \%$ & $15 \%$ & $34.4 \%$ & $7 \%$ & $4 \%$ & $28.1 \%$ & $14 \%$ \\
\hline Emite juicios & $20 \%$ & $15.6 \%$ & $40 \%$ & $11 \%$ & $12.5 \%$ & $14 \%$ & $69 \%$ & $71 \%$ & $46 \%$ \\
\hline
\end{tabular}


Los estudiantes en el grupo focal manifiestan tener buenos desempeños al momento de reconocer errores en el planteamiento de los autores 0 en sus propios planteamientos:

- Estudiante RCh (revisión documental): "Luego de leer el artículo y volver al foro de exploración, considero que un posible imprecisión o error de mis compañeras asociado a creencias sobre el tema es el hecho de catalogar como una causa de la hipertensión la tenencia de una dieta con altos niveles de sal"

- Estudiante VV (Grupo focal): "es algo como cuando nosotros hacemos eso, y a veces si está mal vemos la opinión de las otras personas o lo que dice el profesor o lo que dice en internet o a veces leemos y nosotras como que vemos que está mal, o no estamos conforme con eso"

Para la categoría de resolución de problemas se observa un comportamiento positivo en la autoevaluación inicial y final, específicamente en el indicador que involucra el uso de principios de investigación (Ver tabla 3).

\begin{tabular}{|c|c|c|c|c|c|c|}
\hline Indicadores & Pre-siempre & Post-siempre & Pre-algunas veces & Post-algunas veces & Pre-nunca & Post-nunca \\
\hline Utiliza principios investigación & $18,8 \%$ & $24,1 \%$ & $59,4 \%$ & $65,5 \%$ & $21,9 \%$ & $10,3 \%$ \\
\hline Analiza desde tres perspectivas & $15,6 \%$ & $10,3 \%$ & $71,9 \%$ & $72,4 \%$ & $12,5 \%$ & $17,2 \%$ \\
\hline Soluciona de tres formas diferentes & $18,8 \%$ & $13,8 \%$ & $62,5 \%$ & $69 \%$ & $18,8 \%$ & $17,2 \%$ \\
\hline Utiliza estrategias aprendidas previamente & $31,3 \%$ & $27,6 \%$ & $59,4 \%$ & $58,6 \%$ & $9,4 \%$ & $13,8 \%$ \\
\hline
\end{tabular}

Sin embargo, en el resto de indicadores de esta categoría, se observa una diferencia negativa. Los estudiantes reconocen no hacer uso de manera frecuente de habilidades como análisis desde tres perspectivas, solución de problemas desde diferentes enfoques y utilización de estrategias de solución aprendidas previamente.

En el grupo focal solo se encontraron dos evidencias de las actividades de evaluación. No obstante, estas solo se relacionan con el indicador "Utiliza principios de investigación" coincidente con los resultados de la autoevaluación de los estudiantes: Estudiante DO (Grupo focal) - "en lo que mayor encontré comodidad fue en la actividad de la vitamina $D$ ya que el profesor nos dio un artículo bastante claro en el cual nos mostraba datos porque era una investigación entonces me pareció mucho más cómodo seguir el método científico"

Esto contrasta con los resultados obtenidos en la revisión documental en los cuales se aprecia un avance positivo en todos los indicadores de la categoría Resolución de problemas (Ver tabla 4):

\begin{tabular}{|c|c|c|c|c|c|c|c|c|c|}
\hline \multirow[b]{3}{*}{ Indicadores } & \multicolumn{9}{|c|}{ Frecuencia del hallazgo } \\
\hline & \multicolumn{3}{|c|}{$\begin{array}{c}\text { Siempre } \\
\mathrm{N}^{\circ} \text { Actividad }\end{array}$} & \multicolumn{3}{|c|}{$\begin{array}{c}\text { Algunas veces } \\
\mathrm{N}^{\circ} \text { Actividad }\end{array}$} & \multicolumn{3}{|c|}{$\begin{array}{c}\text { Nunca } \\
\mathrm{N}^{\circ} \text { Actividad }\end{array}$} \\
\hline & $\mathrm{I}$ & II & III & $\mathrm{I}$ & II & III & $\mathrm{I}$ & II & III \\
\hline Utiliza principios investigación & $31 \%$ & $82.2 \%$ & $82 \%$ & $42 \%$ & $17.8 \%$ & $28 \%$ & $27 \%$ & $0 \%$ & $0 \%$ \\
\hline Analiza desde tres perspectivas & $62 \%$ & $0 \%$ & $72 \%$ & $11 \%$ & $39.3 \%$ & $7 \%$ & $27 \%$ & $60.7 \%$ & $21 \%$ \\
\hline Soluciona de tres formas diferentes & $38 \%$ & $0 \%$ & $72 \%$ & $24 \%$ & $60.7 \%$ & $7 \%$ & $38 \%$ & $39.3 \%$ & $21 \%$ \\
\hline Utiliza estrategias aprendidas & $31 \%$ & $39.3 \%$ & $55 \%$ & $19 \%$ & $53.6 \%$ & $16 \%$ & $50 \%$ & $7.1 \%$ & $29 \%$ \\
\hline
\end{tabular}

Algunos indicadores relacionados con la categoría de resolución de problemas, disminuyen en la frecuencia de aparición, esto puede deberse a que en una de las actividades evaluativas no se hicieron explícitos directamente todos los indicadores (Ver tabla 4). En general, los indicadores que más sobresalen son los relacionados con "principios de investigación" y "solución de tres formas diferentes". 
A continuación se presenta una evidencia identificada en la revisión documental, relacionada con el indicador "utiliza principios de investigación" de la categoría de resolución de problemas: Estudiantes CD, KM y GV (revisión documental) “...Las 146.500 personas se dividen equitativamente en cinco grupos (Cada uno con 29.300 personas), uno de ellos haciendo parte de lo que se considera "Grupo Control", los otros cuatros vendrían tomándose como parte del "Grupo Experimental".

Fue particularmente difícil poder encontrar en las actividades desarrolladas por los estudiantes evidencia del indicador: análisis desde tres (3) perspectivas de un mismo asunto. Sin embargo hubo una evidencia que se muestra a continuación: Estudiante CM (revisión documental): "propongo ampliar el estudio, realizando mayores pruebas de lo planteado, proponiendo leyes o hipótesis, basarse en anteriores estudios y en el funcionamiento del organismo y sugiriendo o exponiendo explicaciones que certifiquen la veracidad de los resultados, o en caso de ya poseer tales bases, deberían expresarlas o hacer mención de ellas para mostrar o convencer de la fiabilidad del estudio."

En la categoría análisis del argumento, los resultados de la autoevaluación en todos los indicadores son positivos (Ver tabla 5). La autopercepción de los estudiantes coincide con lo evidenciado en la revisión documental y el grupo focal donde se muestra un avance significativo en especial en los indicadores: "utiliza argumentos para persuadir", "argumenta en temas controversiales" y "juzga la validez de fuentes consultadas". El comportamiento a lo largo del proceso de evaluación muestra el desarrollo de este tipo de habilidades.

\begin{tabular}{|c|c|c|c|c|c|c|}
\hline \multirow[b]{2}{*}{ Indicadores } & \multirow[t]{2}{*}{ Pre-siempre } & \multirow[t]{2}{*}{ Post-siempre } & \multirow{2}{*}{ Pre-algunas veces } & \multirow{2}{*}{ Post-algunas veces } & \multirow[t]{2}{*}{ Pre-nunca } & \multirow[t]{2}{*}{ Post-nunca } \\
\hline & & & & & & \\
\hline Persuasión & $37,5 \%$ & $31 \%$ & $53,1 \%$ & $69 \%$ & $9,4 \%$ & $0 \%$ \\
\hline Identificación de argumentos & $65,6 \%$ & $58,6 \%$ & $28,1 \%$ & $37,9 \%$ & $6,3 \%$ & $3,4 \%$ \\
\hline Semejanza y diferencias & $37,5 \%$ & $44,8 \%$ & $59,4 \%$ & $51,7 \%$ & $3,1 \%$ & $3,4 \%$ \\
\hline Establece validez de las fuentes & $28,1 \%$ & $17,2 \%$ & $62,5 \%$ & $79,3 \%$ & $9,4 \%$ & $3,4 \%$ \\
\hline
\end{tabular}

En la revisión documental de las actividades evaluativas desarrolladas, se observa un decrecimiento de las evidencias de los indicadores: "Identificación de Argumentos" e "Identificación de semejanzas y diferencias en los planteamientos" (Ver tabla 6).

\begin{tabular}{|c|c|c|c|c|c|c|c|c|c|}
\hline \multirow[b]{4}{*}{ Indicadores } & \multicolumn{9}{|c|}{ Frecuencia del hallazgo } \\
\hline & \multirow{2}{*}{\multicolumn{3}{|c|}{$\begin{array}{c}\text { Siempre } \\
\mathrm{N}^{\circ} \text { Actividad }\end{array}$}} & \multirow{2}{*}{\multicolumn{3}{|c|}{$\begin{array}{c}\text { Algunas veces } \\
\mathrm{N}^{\circ} \text { Actividad }\end{array}$}} & \multirow{2}{*}{\multicolumn{3}{|c|}{$\begin{array}{l}\text { Nunca } \\
V^{\circ} \text { Actividad }\end{array}$}} \\
\hline & & & & & & & & & \\
\hline & 1 & II & III & I & II & III & 1 & II & III \\
\hline Persuasión & $58 \%$ & $27.6 \%$ & $60 \%$ & $15 \%$ & $48.3 \%$ & $27 \%$ & $27 \%$ & $24.1 \%$ & $13 \%$ \\
\hline Identificación de argumentos & $73 \%$ & $28.1 \%$ & $63 \%$ & $8 \%$ & $58.6 \%$ & $31 \%$ & $19 \%$ & $10.3 \%$ & $6 \%$ \\
\hline Semejanzas y diferencias & $43 \%$ & $48.3 \%$ & $54 \%$ & $23 \%$ & $37.9 \%$ & $33 \%$ & $34 \%$ & $12.5 \%$ & $13 \%$ \\
\hline Establece validez de las fuentes & $46 \%$ & $12.5 \%$ & $64 \%$ & $0 \%$ & $55.2 \%$ & $23 \%$ & $54 \%$ & $28.1 \%$ & $13 \%$ \\
\hline
\end{tabular}

A continuación se muestra una evidencia de la revisión documental de los indicadores evaluados para la categoría Análisis del Argumento: Estudiante MJ (revisión documental): "Evaluaría la veracidad de la teoría o fundamentación científica del material analizado bajo la óptica de la genética mendeliana... Entonces podría deducir la autenticidad de los resultados expuestos en el artículo o la mencionada posibilidad de que estos hayan sido producto del azar y asimismo la probabilidad de que lo expuesto en él se encuentre relacionado con una variante genética". 
La categoría Pensamiento desde la Incertidumbre no fue evaluada directamente en las actividades propuestas dentro de la investigación, sin embargo emergió en la revisión documental (Ver tabla 7) y en grupo focal.

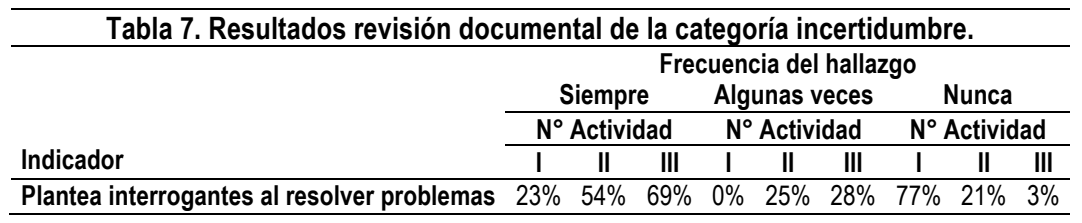

Por último, la categoría Formulación de hipótesis fue evaluada en todas las actividades dado que se incluyeron asignaciones relacionadas con el diseño de experimentos y formulación de hipótesis exigidas por el (M.E.N) en el grado académico que cursan los estudiantes. Cabe anotar que hubo especial dificultad para que algunos estudiantes pudieran formular hipótesis en la forma metodológicamente correcta, aunque para efectos de la investigación la forma de redacción no fue tenida en cuenta pero si su contenido. Como ejemplo se aporta la siguiente evidencia derivada de la revisión documental: Estudiante NP (revisión documental): “ En el ámbito de la genética una hipótesis que encuentro sobre los resultados de esta investigación es que la vitamina $D$ se relacione con las bases del $A D N$, tal vez en su composición haya algo en común que al juntarse mejore muchos aspectos del organismo provocando así que esto mejore las enfermedades cardiovasculares".

\section{Discusión}

La diferencia entre adquirir información y aprender, es el pensamiento crítico. Tal como lo menciona Facione (2007), es posible que exista una relación entre las habilidades del pensamiento crítico y los resultados o desempeños que los estudiantes obtienen en la escuela. Al parecer la relación es directamente proporcional. Facione (2007) también resalta la función del maestro en la promoción del pensamiento crítico quien ya no es la autoridad que todo lo sabe y lo controla. Desde la postura de Valenzuela (2008) se trata de superar la visión memorística hacia un sistema de "aprendizaje profundo" en el cual la comprensión del problema se hace a través de un procesamiento complejo de contenidos.

Uno de los aspectos que identifican al pensador crítico es su capacidad para elaborar y expresar su razonamiento escrito/verbal tal como lo afirman Facione (2007) y Porozo-Méndez (2016). En la revisión de las actividades de evaluación propuestas a los estudiantes se observa un aumento paulatino de evidencias de las categorías que identifican esta habilidad del pensamiento crítico. La lenta progresión de este componente, puede asociase con algunas causas pre existentes en los estudiantes de secundaria y media, tal como reseñan López Silva et al. (2016). Estos autores, identificaron en la autoevaluación que el $64 \%$ de los estudiantes en el área de Biología de la Universidad de Guayaquil presentó un desempeño bajo en este componente. Nuestros resultados en la autoevaluación, aunque no arrojan un resultado tan alto para la baja frecuencia de uso del razonamiento escrito/verbal, si arrojaron un $36,1 \%$ en el segmento de frecuencia de uso moderada (algunas veces). Autores como Villalobos, Ávila y Olivares (2016) consideran que las causas de bajos desempeños en el razonamiento escrito/verbal se deben a la poca adquisición previa de lenguaje científico y/o técnico que incide en la percepción sobre sus desempeños. Otros como Porozo Méndez (2016) afirma que los estudiantes reconocen que no poseen habilidades que les permita resolver problemas de razonamiento verbal/escrito debido a la poca frecuencia de aplicación de ejercicios situados por parte de los docentes. 
Loaiza Zuluaga y Osorio (2018) van más allá y relacionan el lento avance de este componente con el énfasis que colocan los maestros en Colombia en la preparación de los estudiantes a través de pruebas estandarizadas y preguntas de ese mismo corte, descuidando la promoción de la comprensión y la interpretación crítica de textos.

A partir de los resultados, se puede afirmar que la mediación tecnológica (Plataforma Edmodo) pudo ser un factor que facilitó la expresión de los estudiantes y la fluidez al intercambiar opiniones. Esto coincide con lo que expresa Calle Álvarez (2013), quien afirma que en los medios de comunicación virtual se favorece la escritura digital, una estrategia adecuada para desarrollar el pensamiento crítico en los estudiantes. Por su parte, Causado (2015) expresa que el temor a lo que piensen otros compañeros o el miedo a equivocarse se tomaron como responsables del bajo desempeño de los estudiantes en el componente de razonamiento escrito/verbal. Indicadores como la utilización del lenguaje técnico, la emisión de juicios y el reconocimiento de errores son identificados por los estudiantes como de alta dificultad. No obstante, esto contrasta con lo encontrado por los docentes en el grupo focal y la revisión documental en donde se identifican progresos significativos a medida que los estudiantes desarrollan las actividades evaluativas. Lo anterior puede obedecer a un proceso de integración de grupo; un grupo se comporta diferente a la actuación individual dado que en este último los integrantes pueden verse reflejados en el otro, pueden compartir experiencias similares o diferentes en un ambiente de confianza facilitando tanto la correspondencia de visiones sobre una misma realidad (Escobar y Bonilla 2009).

Otra de las categorías del componente de habilidades relacionado con el pensamiento crítico es la resolución de problemas. Según Halpern (1998) todo proceso que involucre el pensamiento crítico, implica tomar decisiones y resolver problemas. Los resultados en esta categoría, señalan que en la autoevaluación los estudiantes consideran tener debilidades con respecto a todos sus indicadores: Utiliza principios de investigación; analiza desde tres perspectivas; soluciona un problema mínimo de tres formas diferentes y utiliza estrategias aprendidas previamente. Esto coincidió con la información del grupo focal.

La información derivada de la revisión documental, por su parte, muestra avances leves, en todos los indicadores de esta categoría. En este punto los resultados encontrados son coincidentes con la investigación de Figueroa y Carrascal (2016), en la cual solo el $14,8 \%$ de los estudiantes alcanzó un nivel superior en este subcomponente del componente de Habilidades. De igual forma, hallazgos de Causado (2015) ponen de manifiesto que en la asignatura de Ciencias Naturales los estudiantes de bachillerato presentan debilidades en el análisis y en el uso de los procesos de investigación en general.

Un aspecto que puede incidir negativamente en los resultados de esta categoría de Habilidades es la forma en la que los docentes usualmente abordan la resolución de problemas en la enseñanza de las ciencias naturales. En este punto Jessup (2017), resalta que los problemas a los que está acostumbrado el estudiantado son simples ejercicios llevados a la categoría de problemas, lo que lleva a este a una habituación e incapacidad de solucionar problemas. Asimismo, Lara, Ávila y Olivares (2017), aportan que la escasa exposición de oportunidades relacionadas con solución de problemas trae consigo que esta no sirva como potenciadora del pensamiento crítico, por lo que se recomienda que sea continúa la implementación de estrategias para este fin.

Cabe anotar que el único indicador de esta categoría que presentó incremento en la autoevaluación y en la revisión documental fue el de uso de principios de investigación, quizá por tratarse de una asignatura como la Biología en la cual es 
fundamental tener en cuenta los principios del método científico. Vázquez-Alonso y Manassero (2017), afirman que es mejor enseñar para comprender cómo funciona la ciencia, que enseñar para saber ciencias. Esto es reforzado desde la postura de Halpern (2006) quien sostiene que la educación desde la ciencia, su método y sus procedimientos favorecen al futuro científico y también al que se convertirá en consumidor de ciencia, dado que incluso en lo cotidiano estamos inmersos en información científica y no científica que pueden ser abordadas en nuestra vida usando los principios científicos. Ay, Karakaya y Yilmaz (2015) y Cepeda, Cordova y Cabezas (2017), plantean que los pensadores críticos deben tener características propias de los científicos, por lo cual los principios de investigación deben ser guía fundamental de la actuación del docente.

En la categoría análisis del argumento, los resultados de la autoevaluación en todos los indicadores resultaron positivos. La autopercepción de los estudiantes coincide con lo evidenciado en la revisión documental y en el grupo focal. Estos resultados son diferentes y entran en contraposición con los resultados de Figueroa y Carrascal (2016) quienes también usaron los componentes de Halpern (1998). Sus resultados arrojan un bajo porcentaje $(40,7 \%)$ de estudiantes con niveles superior y alto para esta misma categoría, en comparación con los resultados de la presente investigación que presentó un $60,25 \%$ en frecuencia alta en la revisión documental realizada a las actividades evaluativas. Los resultados de Figueroa y Carrascal (2016) coinciden con lo que exponen Villalobos et al. (2016), sobre los problemas para argumentar y describir de forma coherente y para emitir opiniones y juicios por parte de los estudiantes de bachillerato. Autores como Loaiza Zuluaga y Osorio (2018) atribuyen los resultados bajos en el análisis del argumento a los cambios psico-biológicos propios de la transición adolescente que pueden afectar algunos criterios de inferencia y argumentación.

Como categoría emergente se identificó el pensamiento desde la Incertidumbre, pues aunque no se evaluó de forma directa, se reflejaron hallazgos de ella en los resultados de la revisión documental y en el grupo focal. Halpern (2014) incluye de manera conjunta la habilidad de pensar bajo principios de incertidumbre, como inherente al proceso de pensamiento crítico desde la cual el pensador crítico asume y evalúa desde cierta perspectiva los riesgos, dudas y temores en el momento de resolver problemas. Esto demuestra que las habilidades de pensamiento crítico así como los componentes del mismo se articulan de forma transversal y es posible que se evidencien aun cuando explícitamente no estén siendo evaluadas.

\section{Conclusión}

En concreto, los resultados muestran una clara tendencia: las actividades de evaluación formativa del pensamiento crítico propuestas a través de la mediación tecnológica EDMODO posibilitan las habilidades del pensamiento crítico de los estudiantes de educación media. Esta tendencia se confirma con los resultados que corresponden a una alta frecuencia de evidencias en las categorías del componente habilidades del pensamiento crítico propuestas por Halpern $(2003 ; 2006)$ y adaptadas a nuestro estudio. La mediación tecnológica parece ser efectiva en favorecer las habilidades del pensamiento crítico en la medida que al expresarse a través de una vía digital, los estudiantes que presentan temor o inseguridad para utilizar el lenguaje hablado pueden ejercitar su argumentación o juzgar la de otros de una forma más cómoda. En la presente investigación se evidencia que desde la biología se puede facilitar la activación de procesos complejos de pensamiento y que la mediación con EDMODO, sirve a este propósito. 
De acuerdo a las evidencias relacionadas con el desarrollo paulatino del pensamiento crítico, estos resultados han de tomarse con precaución pues el presente trabajo tiene en cuenta sólo un caso, con un número reducido de participantes por lo que sus conclusiones pueden entenderse como parciales, no generalizable. No obstante, resulta ser útil como una vía para la promoción de este tipo de pensamiento. La información recogida y el análisis de esta, permiten ofrecer información sobre la importancia de considerar el desarrollo del pensamiento crítico como un eje transversal no solo en lo académico sino para la vida. En nuestra investigación el acompañamiento docente fue fundamental tal como se registra en las evidencias del grupo focal.

Está claro que el pensamiento crítico puede ser transferido y promocionado dentro del aula o a través de diversos escenarios articulados a esta como las TIC. Estas también permiten al estudiante aumentar el aprendizaje y las habilidades cuando son usadas como ejes de la actividad cognitiva, colaborativa y comunicativa, así mismo las plataformas digitales pueden servir para compartir productos intelectuales de los estudiantes Muñoz-Repiso y Tejedor (2017).

La utilización de las TIC fue percibida por el alumnado como favorable, se convierten en mediadoras y posibilitan confrontar información y hechos, sin moverse de un sitio.

No queremos finalizar sin mencionar que es necesario incluir en las dinámicas del aula el diálogo, las discusiones y la toma conjunta de decisiones en torno a las actividades escolares, a la selección y organización del conocimiento que se propone aprende. Las mediación pedagógica de las TIC, permiten democratizar la educación brindando oportunidades para fortalecer las habilidades del pensamiento crítico de los estudiantes.

\section{Referencias}

Akyüz, H.., Samsa Yetik, S., \& Keser, H. (2015). Effects of Metacognitive Guidance on Critical Thinking Disposition. Online Submission, 5(2), 133-148.

Ay, FA., Karakaya, A., \& Yilmaz, K. (2015). Relations between selfleadership and critical thinking skills. Procedia-social and Behavioral sciences, 207, 29-41.

Bailin, S., Case, R., Coombs, JR., \& Daniels, LB. (1999). Conceptualizing critical thinking. Journal of curriculum studies, 31(3), 285-302.

Bellanca, J., \& Brandt, R. (2010). 21st Century Skills: Rethinking How Students Learn. Bloomington: Solution Tree Press.

Calle Álvarez, G. (2013). La evaluación de las habilidades del pensamiento crítico asociadas a la escritura digital. Revista Virtual Universidad Católica del Norte, 3(40), 68-83.

Calle Álvarez, G. (2014). Las habilidades del pensamiento crítico durante la escritura digital en un ambiente de aprendizaje apoyado por herramientas de la web 2.0. Revista Encuentros, Universidad Autónoma del Caribe, 12(1), 27-45.

Cano Rodríguez, GM. y González Guzmán, J. (2017). ConTIC aprendí: Aula invertida como modelo para promover el pensamiento crítico en estudiantes de grado noveno del Colegio Antonio García. Tesis de Maestría de la Universidad de La Sabana, Bogotá, Colombia.
Causado, R. (2015). Desarrollo del pensamiento critico en el área de Ciencias Naturales en una escuela de secundaria. Revista de la Facultad de Ciencias,4(2), 17-42.

Cepeda, MP., Cordova, RMZ. y Cabezas, JAA. (2017). Pensamiento crítico aplicado a la investigación científica. Revista Atlante: Cuadernos de Educación y Desarrollo. [Archived by WebCite ${ }^{8}$ at http://www.webcitation.org/74pkl0vyU].

DeNoyelles, A. y Reyes-Foster, B. (2015). Using word clouds in online discussions to support critical thinking and engagement. Online Learning, 19(4).

Díaz-Barriga, F. (2001). Habilidades de pensamiento crítico sobre contenidos históricos en alumnos de bachillerato. Revista Mexicana de Investigación Educativa. [Archived by WebCite® at http://www.webcitation.org/74pkxwHXg].

Drewett, M. (1995). There must be some way out of here: a case study in the teaching of critical thinking. South African journal of philosophy, 14(2), 72-76.

Duque, W. (2015). Nuevas dinámicas pedagógicas en redes sociales: Aprovechamiento tecnológico de los grupos cerrados de Facebook para potenciar los procesos de aprendizaje en el área de tecnología e informática del grado 11 de la IE Joaquín Cárdenas Gómez (tesis de maestría). Universidad Pontificia Bolivariana, Medellín, Colombia. 
Duran, M., \& Sendag, S. (2012). A preliminary investigation into critical thinking skills of urban high school students: role of an IT/STEM program. Creative Education, 3(2), 241-250. http://dx.doi.org/10.4236/ce.2012.32038

Ennis, RH. (1993). Critical thinking assessment. Theory Intro Practice, 32(3), 179-186.

Ennis, RH., \& Weir, EE. (1985). The Ennis-Weir critical thinking essay test: An instrument for teaching and testing. Cheltenham: Hawker Brownlow.

Ennis, RH., Gardiner, WL., Guzzetta, J., Morrow, R., Paulus, D., \& Ringel, L. (1964). The Cornell Conditional-Reasoning Test, Form X. Ithaca: Cornell Critical Thinking Project. [Archived by WebCite $®$ at http://www.webcitation.org/74pzCYkCX]

Escobar, J. y Bonilla-Jimenez, FI. (2009). Grupos focales: una guía conceptual y metodológica. Cuadernos hispanoamericanos de psicología, 9(1), 51-67.

Facione, P. (2007). Pensamiento Crítico: ¿Qué es y por qué es importante?. Insight assessment, 23, 56. [Archived by WebCite $₫$ at http://www.webcitation.org/74uSiqNsM]

Facione, PA., Facione, NC. y Giancarlo, CAF. (2001). California critical thinking disposition inventory: CCTDI. Mellbrae: California Academic Press.

Facione, PA., Facione, NC., Blohm, SW., \& Gittens, CA. (1990). The California critical thinking skills test manual. Mellbrae: California Academic Press.

Figueroa, HAG. y Carrascal, AA. (2016). Potencialización del pensamiento crítico a través de la hipotemetacomprensión textual (HMT). Cultura Educación y Sociedad, 7(2), 54-71.

Finken, M., \& Ennis, RH. (1993). Illinois Critical Thinking Essay Test. Department of Educational Policy Studies, Champaign, IL. [Archived by WebCite ${ }^{\circ}$ at
http://www.webcitation.org/74uSDW7HU]

Halpern, DF. (1998). Teaching critical thinking for transfer across domains: Disposition, skills, structure training, and metacognitive monitoring. American psychologist, 53(4), 449.

Halpern, DF. (2003). Thinking: An Introduction in Thought and knowledge: An introduction to critical thinking. New York: Psychology Press.

Halpern, DF. (2006). "The nature and nurture of critical thinking" in Robert Sternberg, Henry Roediger y Diane Halpern (eds.): Critical thinking in psychology (1-14). Cambridge: Cambridge University Press.

Halpern, DF. (2014). Critical thinking across the curriculum: A brief edition of thought and knowledge. Mahwah: Lawrence Erlbaum Associates Publishers.

Jessup, MNJC. (1998). Resolución de problemas y enseñanza de las ciencias naturales. TED: Tecné, Episteme y Didaxis, artículo número 3. doi.org/10.17227/ted.num3-5701

Lara Quintero, V., Avila Palet, JE. y Olivares Olivares, SL. (2017). Desarrollo del pensamiento crítico mediante la aplicación del Aprendizaje Basado en Problemas. Psicología Escolar e Educacional, 21(1), 65-77.
Lipman, M. (1987). Critical thinking: What can it be?. Analytic Teaching, 8(1), 5-7.

Loaiza Zuluaga, Y. y Osorio, L. (2018). El desarrollo de pensamiento crítico en ciencias naturales con estudiantes de básica secundaria en una Institución Educativa de PereiraRisaralda. Diálogos sobre educación. Temas actuales en investigación educativa, 9(16), 1-24.

López Silva, BO., García, R., Hernández Navarro, Ml., López Córdova, BA., López Córdova, MO. y Barbies Rubiera, A. (2016). El pensamiento crítico-analítico en estudiantes del área de Biología de la Universidad de Guayaquil, Ecuador. Edumecentro, 8(3), 38-51.

López, G. (2012). Pensamiento crítico en el aula. Docencia e Investigación, 37(22), 41-60.

Manassero-Mas, MA. y Vázquez-Alonso, Á. (2017). ¿Hay contenidos de naturaleza de la ciencia y la tecnología y pensamiento crítico en los currículos (españoles) actuales?. Enseñanza de las ciencias: revista de investigación y experiencias didácticas, Número extra: $\mathrm{X}$ Congreso Internacional sobre Investigación en Didáctica de las Ciencias, 509-514.

Ministerio de educación nacional de Colombia - M.E.N. (2004). Estándares Básicos de Competencias en Ciencias naturales y Ciencias sociales. Bogotá: Imprenta Nacional de Colombia. [en línea]. [Archived by WebCite® at http://www.webcitation.org/74q0YhpWJ].

Muñoz-Repiso, AGV. y Tejedor, FJT. (2017). Percepción de los estudiantes sobre el valor de las TIC en sus estrategias de aprendizaje y su relación con el rendimiento. Educación XX1, 20(2), 137-159.

Olivares, SL. y Heredia, Y. (2012). Desarrollo del pensamiento crítico en ambientes de Aprendizaje basado en problemas en estudiantes de educación superior. Revista Mexicana de Investigación Educativa, 17(54), 759-778.

Paul, R. y Elder, L. (2003). Estándares de competencia para el pensamiento crítico. Fundación para el pensamiento crítico. [Archived by WebCite $\AA$ at http://www.webcitation.org/74q0obStz].

Porozo Méndez, C. (2016). Desarrollo del razonamiento verbal como estrategia didáctica para la comprensión del texto (Tesis de maestría). Pontificia Universidad Católica del Ecuador -PUCESE-, Esmeraldas, Ecuador.

Robles, H. y Rodríguez, R. (2013). Un ambiente virtual para las habilidades de pensamiento crítico en ESL. Zona Próxima, 19, 73-85.

Rumpagaporn, MW. (2007). Student's critical thinking skills, attitudes to ICT and perceptions of ICT classroom learning environment under the ICT schools pilot project in Thailand. School of Education, Bangkok: University of Adelaide.

Sá, CM. (2016). Pensamento crítico, TIC e formação em didática de línguas. Revista Lusófona de Educação, 32, 93-107.

Saiz, C. y Rivas, S. (2008). Intervenir para transferir en pensamiento crítico. Praxis, 10(13), 129-149. 
Severin, E. (2011). Competencias para el siglo XXI: cómo medirlas y cómo enseñarlas. División de Educación del Banco Interamericano de Desarrollo BID. Washington, DC 20577

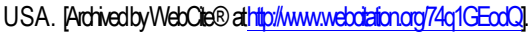

Siegel, H. (1985). Educating reason: Critical thinking, informal logic, and the philosophy of education. Informal Logic, 7(2), 69-81.

Stake, RE. (2007). Investigación con estudio de casos. Ediciones Morata.

Thompson, A. y Crompton, H. (2010). Point/Counterpoint is technology killing critical thinking? Learning and Leading with Technology, 38(1), 6.

Valenzuela, J. (2008). Habilidades de pensamiento y aprendizaje profundo. Revista Iberoamericana de Educación, 46(7), 1-9.

Valenzuela, J. y Nieto, AM. (2010). Motivación y disposiciones como predictores del desempeño del pensamiento crítico. En JM. Díaz Gómez y E. Gámez Armas E. (coord.), Motivación y emociones (135-150). San Critobal de La Laguna: Editorial Universidad de la Laguna.

Vásquez Cano, E. (2013). Microblogging con Edmodo para el desarrollo de las competencias básicas del alumnado de enseñanza secundaria. Un estudio de caso. Educación Siglo XXI, 31(1), 313-334.

Villalobos Delgado, V., Ávila Palet, JE, y Olivares, S. (2016). Aprendizaje basado en problemas en química y el pensamiento crítico en secundaria. Revista mexicana de investigación educativa, 21(69), 557-581.

Wichadee, S. (2014). Students' Learning Behavior, Motivation and Critical Thinking in Learning Management Systems. Journal of Educators Online, 11(3), 1-21. 